\title{
A CRITERION FOR STABLE CHARACTERISTIC EXPONENTS*
}

\section{BY AUREL WINTNER (The Johns Hopkins University)}

1. Let $f(t)$ be a real-valued, continuous, periodic function and let the unit of length on the $t$-axis be so chosen that the period of $f(t)$ becomes 1 . Then the linear differential equation

$$
x^{\prime \prime}+f(t) x=0
$$

has two solutions of the form

$$
x=e^{\lambda t} g(t), \quad x=e^{-\lambda t} \hat{h(t),}
$$

where $g(t)$ and $h(t)$ have the period, 1 , of $f^{\prime}(t)$ and do not vanish identically. The numbers $\pm \lambda$ occurring in (2), the characteristic exponents of (1), are determined mod $2 \pi i$ only. If $\lambda$ is not a multiple of $\pi i$, it is clear that the two solutions (2) are linearly independent. If $\lambda$ is a multiple of $\pi i$, it depends on the elementary divisors of certain linear substitutions whether (1) does or does not possess two linearly independent solutions of the form (2) (in the second case, the general solution of (1) contains a secular term, that is, a term containing the factor $t$ ).

Let the differential equation (1) be called of stable type if every solution, $x(t)$, remains bounded as $t \rightarrow \pm \infty$, and let the characteristic exponents $\pm \lambda$ of (1) be called of stable type if they are purely imaginary (including 0 ). Then it is clear that (1) cannot be of stable type unless the characteristic exponents are, and that (1) must be of stable type if the characteristic exponents are of stable type and distinct. Needless to say, the two characteristic exponents, $\lambda$ and $-\lambda$, are considered as distinct if and only if they are distinct $\bmod 2 \pi i$ (that is, if $\lambda$ is not a multiple of $\pi i$ ).

2. The usual way of calculating $\lambda$ is supplied by Hill's method of infinite determinants (the zeros of the resulting transcendental equation being all possible values,

$$
\pm \lambda, \quad \pm \lambda \pm 2 \pi i, \quad \pm \lambda \pm 4 \pi i, \cdots,
$$

of the characteristic exponent). For certain purposes, a less elaborate procedure is more convenient. Quite a direct procedure happens to be contained in the classical proof (Cauchy-Fuchs-Floquet) for the mere existence of solutions of the form (2). In fact, this existence proof is based on the consideration of the linear substitutions of the "monodromy group." These are binary substitutions of determinant 1 , have a common pair of latent roots, and the logarithms of these roots are precisely the characteristic exponents. In other words, $e^{ \pm \lambda}$ is the root of a reciprocal quadratic equation, say

$$
e^{ \pm 2 \lambda}-2 \alpha e^{ \pm \lambda}+1=0,
$$

where $\alpha$ is a constant uniquely determined by the periodic coefficient function of (1).

Accordingly, the calculation of $\lambda$ can be reduced to the calculation of $\alpha$. But $\alpha$ can be calculated from $f(t)$, by applying the method of successive approximations to those two solutions of (1) which are determined by the initial conditions

\footnotetext{
* Received March 12, 1947.
} 


$$
x(0)=1, \quad x^{\prime}(0)=0 \text {. and } x(0)=0, \quad x^{\prime}(0)=1 .
$$

This procedure, which seems to go back to Liapounoff (cf. pp. 425-431 of vol. IV, part III (1902) of Forsyth's Theory of Differential Equations), leads to the following representation of $\alpha$ :

Put

$$
F(t)=\int_{0}^{t} f\left(t_{0}\right) d t_{0}
$$

and

$$
F_{n}=\left\{\dot{F}(1)-F\left(t_{1}\right)+F\left(t_{n}\right)\right\} \prod_{k=1}^{n-1}\left\{F\left(t_{k}\right)-F\left(t_{k+1}\right)\right\},
$$

where $n=1,2, \cdots$ (with the understanding that

$$
\prod_{k=1}^{n-1}=1 \quad \text { if } \quad n=1
$$

that is, if the product is vacuous); so that

$$
F_{n}=F_{n}\left(t_{1}, \cdots, t_{n}\right) .
$$

Then, if the constants $\alpha_{1}, \alpha_{2}, \cdots$ are defined by

$$
\alpha_{n}=\frac{1}{2} \int_{0}^{1} d t_{1} \int_{0}^{t_{1}} d t_{2} \cdots \int_{0}^{t_{n-1}} F_{n}\left(t_{1}, \cdots, t_{n}\right) d t_{n}
$$

(that is, if the integral of (5) over the $n$-dimensional polytope

$$
0 \leqq t_{n} \leqq t_{n-1} \leqq \cdots \leqq t_{1} \leqq 1
$$

is denoted by $2 \alpha_{n}$ ), the series

$$
\alpha=1-\alpha_{1}+\alpha_{2}-\cdots+(-1)^{k} \alpha_{k}+\cdots
$$

is convergent and represents the coefficient in (3). This is the substance of the verifications which will be found loc. cit.

This method does not seem to be generally known among applied mathematicians; it is not mentioned in Strutt's usually quite complete monograph.

3. Since (6), hence (8), is real-valued, and since the roots of the quadratic equation (3) are

$$
e^{ \pm \lambda}=\alpha \pm\left(\alpha^{2}-1\right)^{1 / 2},
$$

it is clear that the characteristic exponents $\pm \lambda$ are of stable type (that is, purely imaginary, including 0 ) if and only if

$$
-1 \leqq \alpha \leqq 1,
$$

and that they are of stable type and distinct $(\bmod 2 \pi i)$ if and only if

$$
-1<\alpha<1 \text {. }
$$


Hence, the stability of (1) depends on whether the sum of the numerical series (8) does or does not satisfy these conditions.

Liapounoff has used this method in order to show that, if $f(t)=f(t+1)$ is positive throughout, the characteristic exponents of (1) must be of stable type whenever the average value of $f(t)$, that is, the constant term in the Fourier expansion of $f(t)$, is sufficiently small; namely, less than 4 (cf. loc. cit.). Liapounoff's proof can be interpreted as amounting to an application of the second mean-value theorem in integral calculus. By using the first mean-value theorem instead of the second, two stability criteria of quite another type will be deduced in the sequel. Neither of them will assume that $f(t) \geqq 0$. The first of them is as follows:

(i) Let $f(t)$ be a real-valued, continuous function of period 1. Suppose that its average

$$
\mu=\int_{0}^{1} f(t) d t
$$

and its absolute maximum

$$
M=\max |f(t)|
$$

satisfy the inequalities

$$
\sum_{n=2}^{\infty} M^{n} /(2 n-1) !<\mu \leqq 1
$$

(which are compatible with $\min f(t)<0$ ); for instance, that

$$
M^{2} e^{M}<6 \mu \leqq 6 .
$$

Then the characteristic exponents of (1) are of stable type and distinct.

That (13) is sufficient for (12), is seen by comparing coefficients.

As to the possibility mentioned parenthetically after (12), it is sufficient to observe that, while (11) and (12) imply that

$$
\mu>0,
$$

(14) and (10) do not imply that $f(t)$ is non-negative throughout.

4. According to (4), the first factor on the right of (5) is identical with the difference

$$
\int_{0}^{1} f(t) d t-\int_{t_{n}}^{t_{1}} f(t) d t
$$

where $0 \leqq t_{n} \leqq t_{1} \leqq 1$. It follows therefore from (11) and (5) that

$$
\left|F_{n}\right| \leqq M \prod_{k=1}^{n-1}\left|F\left(t_{k}\right)-F\left(t_{k+1}\right)\right| \text {. }
$$

On the other hand, from (4) and (11),

$$
\left|F\left(t_{k}\right)-F\left(t_{k+1}\right)\right| \leqq M\left(t_{k}-t_{k+1}\right),
$$

where $t_{k+1} \leqq t_{k}$, by (7). Hence, the inequality

$$
\left|F_{n}\right| \leqq M^{n} D_{n}
$$


holds at every point of the polytype (7), if $D_{n}=D_{n}\left(t_{1}, \cdots, t_{n}\right)$ is an abbreviation for the product

$$
D_{n}=\prod_{k=1}^{n-1}\left(t_{k}-t_{k+1}\right),
$$

the (non-negative) square-root of the discriminant of the polynomial $\prod_{k=1}^{n}\left(z-t_{k}\right)$.

The integral of the product (16) over the polytope (7) is known to have the value $1 /(2 n-1)$ !. It follows therefore from (6) and (15) that

$$
\left|\alpha_{n}\right| \leqq \frac{1}{2} M^{n} /(2 n-1) ! \text {. }
$$

Hence, by the first of the inequalities (12),

$$
\sum_{n=2}^{\infty}\left|\alpha_{n}\right|<\frac{1}{2} \mu \text {. }
$$

According to (5) and the parenthetical remark which follows (5), the function $F_{1}$ is identical with the constant $F(1)$. It follows therefore from (6) that $\alpha_{1}=\frac{1}{2} F(1)$. In view of (4) and (10), this means that

$$
\alpha_{1}=\frac{1}{2} \mu \text {. }
$$

Consequently, from (8) and (18),

$$
\left|\alpha-\left(1-\frac{1}{2} \mu\right)\right|<\frac{1}{2} \mu \text {. }
$$

In view of the second of the inequalities (12), the last formula line implies that

$$
0<\alpha<1 \text {. }
$$

Since this, in turn, implies that (9) is satisfied, the proof is complete.

The first of the assumptions (12) can. be improved somewhat. In fact, it is clear from the proof of (15) that (15) can be refined to

$$
\left|F_{n}\right| \leqq M^{n} D_{n}-M^{n}\left(t_{1}-t_{n}\right) D_{n},
$$

where $\left(t_{1}-t_{n}\right) D_{n}$ is positive (within the polytope).

5. Since only (9) was needed but (20) was deduced, it is natural to ask after a condition which, in contrast to (12), leads to the $\alpha$-range complementary to (21), that is, to the range

$$
-1<\alpha \leqq 0 \text {. }
$$

Such a condition is contained in the following dual of (i):

(ii) The pair of assumptions, (12), of (i) can be replaced by

$$
\sum_{n=2}^{\infty} M^{n} /(2 n-1) !<4-\mu \leqq 2 ;
$$

for instance, (13) can be replaced by

$$
M^{2} e^{M}<24-6 \mu \leqq 12 .
$$

In fact, (17) and (19) were obtained without any hypothesis and are therefore applicable whether (12) is assumed or not. But (17), (19) and (8) imply that 


$$
|\alpha| \leqq\left|1-\frac{1}{2} \mu\right|+\frac{1}{2} \sum_{n=2}^{\infty} M^{n} /(2 n-1) !
$$

Hence, by the first of the inequalities (23),

$$
|\alpha|<\left|1-\frac{1}{2} \mu\right|+2-\frac{1}{2} \mu \text {. }
$$

On the other hand, the second of the inequalities (23) can be written in the form $\mu \geqq 2$, which means that

$$
\left|1-\frac{1}{2} \mu\right|=\frac{1}{2} \mu-1 \text {. }
$$

This completes the proof, since the last two formula lines imply the inequality $|\alpha|<1$, which is (9).

Conclusion. If $\mu, M$ are defined by (10), (11), then either (12) or (23) [and so, in particular, either (13) or (24)] is sufficient for stability.

As an illustration, let

$$
f(t)=(a+b \cos 2 \pi t)^{-1}, \text { where } 0<b<a ;
$$

so that (1) becomes the equation known from the problem of frequency modulation. In this case, (10) and (11) reduce to

$$
\mu=\left(a^{2}-b^{2}\right)^{-1 / 2} \text { and } M=(a-b)^{-1},
$$

and so the above inequalities supply explicit conditions for pairs $(a, b)$ which are sure to be of stable type. Needless to say, the resulting inequalities for $a$ and $b$ are just sufficient for stability. Incidentally, since $f(t)$ is now positive, Liapounoff's criterion, $\mu<4$, also is applicable.

\section{LOWER BUCKLING LOAD IN THE NON-LINEAR BUCKLING THEORY FOR THIN SHELLS*}

\section{By HSUE-SHEN TSIEN (Massachusetts Institute of Technology)}

For thin shells the relation between the load $P$ and the deflection $\epsilon$ beyond the classical buckling, load is very of ten non-linear. For instance, when a uniform thin circular cylinder is loaded in the axial direction, the load $P$ when plotted against the end-shortening $\epsilon$ has the characteristic shown in Fig. 1. If the strain energy $S$ and the total potential $\varphi=S-P \epsilon$ are calculated, their behavior can be represented by the curves shown in Figs. 2 and 3. It can be demonstrated that the branches $O C$ and $A B$ corresponds to stable equilibrium configurations and the branch $B C$ to unstable equilibrium configurations. The point $B$ is then the point of transition from stable to unstable equilibrium configurations.

It was proposed by the author in a previous paper ${ }^{1}$ that the point $A$ was the critical point for buckling of the structure under external disturbances, using the $S, \epsilon$ curve for "testing machine" loading and the $\varphi, P$ curve for "deadweight" loading. The load $P$ for the unbuckled configuration of the shell corresponding to the point $A$ was called

* Received April 2, 1947.

${ }^{1} \mathrm{H}$. S. Tsien, $A$ theory for the buckling of thin shells, J. Aero. Sciences 9, 373-384 (1942). 